\title{
The Link between Early Adolescent Alcohol Abuse and Adult Antisocial Behaviour: A Hypothesis Revisited
}

\author{
Richard C. Howard \\ Institute of Mental Health Nottingham, UK
}

Received: September 3, 2016; Accepted: September 5, 2016; Published: September 13, 2016

*Corresponding author: Richard C. Howard PhD, Institute of Mental Health, Nottingham, UK, Tel: +49-611-527-264; E-mail: richard.howard@nottingham.ac.uk

\begin{abstract}
Ten years ago the author advanced a novel hypothesis that proposed a causal link between childhood conduct disorder (CD) and early alcohol abuse in the genesis of adult antisocial behavior. In the context of antecedent $\mathrm{CD}$, progressive and accelerating use of alcoholand other drugs in adolescence was said to result in increasing disinhibition and progressive misuse of alcohol. Exposure of the vulnerable adolescent brain to excessive amounts of alcohol and other substances putatively results in structural and functional changes in the brain, particularly in those areas involved in emotional selfregulation. As a consequence, such individuals emerge into adulthood as emotionally impulsive and at high risk of serious antisocial conduct.

Evidence is here reviewed that supports several propositions. arising from this hypothesis: 1 . The transition from childhood conduct disorder (CD) to adult antisocial behavior is mediated and moderated by early alcohol abuse. 2 . Emotional impulsiveness is a core feature of severe personality disorder. 3. Severe personality disorder and emotional impulsiveness are associated with severe criminal violence. 4. Reoffending in patients with personality disorders is associated with severe drug and alcohol abuse, adult antisocial personality with severe $\mathrm{CD}$, and severe borderline personality disorder. 5. Emotional impulsiveness as seen in severe personality disorder commonly manifests in anger. 6 . An emotionally impulsive brain can be trained to be less impulsive.
\end{abstract}

Keywords: Alcohol; Substance Abuse; Impulsiveness; Personality Disorder; Violence; Antisocial; Borderline

\section{Introduction}

Some 10 years ago the author advanced the hypothesis that early-onset alcohol abuse might play a critical role in the aetiology of adult antisocial behaviour [1]. This hypothesis had its origins in a chance observation that an electrophysiological correlate of emotional impulsiveness, the Go/No Go contingent negative variation (CNV: Figure 1), showed a "high impulsive" pattern in those prison inmates who had a history of early-onset alcohol abuse, compared with inmates who lacked such a history [2]. Go/No Go CNV had previously been shown to reliably predict both general and violent reoffending in patients with personality disorder who had been discharged from high-secure care into the community $[3,4]$. Thus it was reasonable to assume that emotional impulsiveness - the tendency to act precipitately and without consideration of adverse consequences under pressure of negative emotions such as anger - might be a core feature of severe personality disorder, one that was associated with a high risk of violent offending. Both childhood CD [5] and early alcohol abuse [6, 7], particularly in the form of binge drinking [8] have independently been implicated as precursors of later antisocial behavior, and of violence in particular [8]. However, a variable proportion of those children diagnosed with $\mathrm{CD}$ have been found to progress to antisocial personality disorder as adults estimates vary across different samples between $30 \%[9,10]$ and $60 \%$ [11]. Howard's hypothesis was novel in explicitly proposing a causal link between CD and early alcohol abuse in the genesis

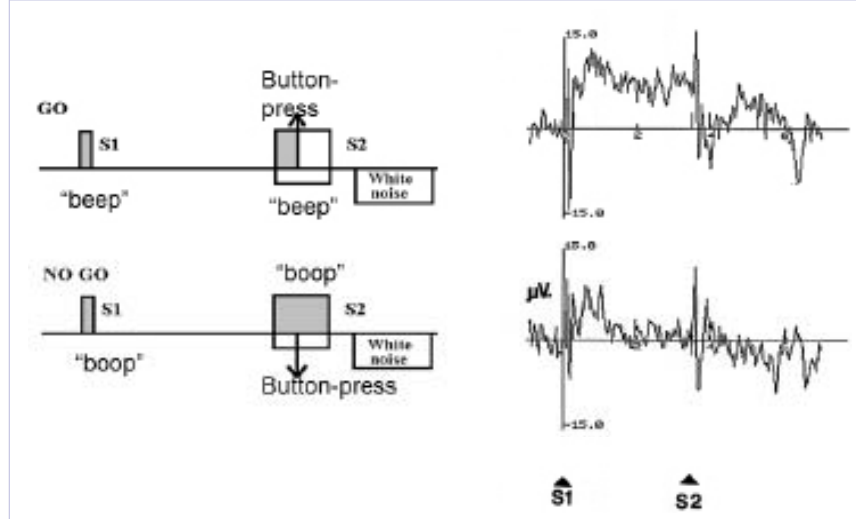

Figure 1: Go/No Go Contingent Negative Variation (CNV). The left side of the figure shows the behavioral paradigm for eliciting the Go CNV (upper trace on right of figure) and No Go CNV (lower trace on right of figure). The difference between Go and No Go wave forms reflects emotional impulsiveness: emotionally impulsive individuals show a lack of differentiation between Go and No Go waveforms. Go and No Go trials (16 of each) are randomly interleaved. On Go trials, a tone "beep" (S1) signals that on receipt of a second "beep" (S2) $3 \mathrm{~s}$. later, a rapid buttonpress response is required to avoid an aversive white noise. On No Go trials, a "boop" (S1) signals that on receipt of a second "boop" (S2) $3 \mathrm{~s}$. later, a button-press results in receipt of white noise - the subject must refrain from pressing to avoid the white noise. 
of adult antisocial behavior. Specifically, CD was said initially to lead to a progressive and accelerating use of alcohol and other drugs in adolescence. A vicious cycle then develops whereby increasing use of alcohol in adolescence results in increasing disinhibition and progressive misuse of alcohol. Exposure of the vulnerable adolescent brain to excessive amounts of alcohol and other substances putatively results in structural and functional changes in the brain, particularly in those prefrontal areas involved in emotional and behavioural self-regulation. As a consequence, on entering adulthood such individuals suffer deficits in self-regulation that place them at high risk of serious antisocial conduct.

Howard's hypothesis was later elaborated [12] in the context of a "dual systems" model of adolescent decision making and development proposed by Steinberg and colleagues [13]. Support for this model has accrued over the years since it was first proposed (reviewed in [14]). In short, it proposes" that risk-taking behaviors peak during adolescence because activation of an earlymaturing incentive-processing system (the "socio emotional system") amplifies adolescents' affinity for exciting, novel and risky activities, while a countervailing, but slower to mature, "cognitive control" system is not yet far along in its development to consistently restrain potentially hazardous impulses" [14, p. 104]. In elaborating Howard's model, Howard \& Mc Murran [12]proposed that excessive use of alcohol, and binge drinking in particular, in young (roughly 13-15 year-old) adolescents resulted from an over-sensitive socio emotional system in a proportion of those diagnosed with CD, particularly those showing "callous and unemotional" traits. Critically, this early abuse of alcohol was said to impair and/or delay development of the cognitive control system (and its neural substrates in lateral prefrontal cortex) whose normal development proceeds in a slow and linear fashion during adolescence and continues into early adulthood. Disrupted development of the cognitive control system will then result, during late teens and early twenties, in a chronic inability to control emotional impulses and hence a proneness to engage in violence.

Howard's hypothesis generated several sub-hypotheses that have in general been supported by subsequent research, to be reviewed below.

\section{The transition from childhood conduct disorder (CD) to adult antisocial behavior is mediated and moderated by early alcohol abuse}

This hypothesis was tested in two studies, one examining a North American sample of 504 young men and women recruited from the community [15], the other a sample of 100 personality disordered male offenders detained in an English high-secure hospital [16]. The first study tested the prediction that those with a history of both childhood CD and early alcohol abuse/dependency would show a significantly higher degree of antisocial behavior in late adolescence and early adulthood when compared with those who showed CD alone. This hypothesis was confirmed. Regression analyses showed that a history of alcohol abuse/dependence both moderated (i.e. exacerbated) and partially mediated the relationship between $\mathrm{CD}$ and adult antisocial behavior.

The second study [16] showed that those offenders in whom CD co-occurred with early-onset alcohol abuse showed the greatest severity of personality pathology, particularly in terms of Cluster B traits and antisocial/borderline PD comorbidity; they also showed more violence in their criminal history compared with those showing only CD. Regression analysis showed that both early alcohol abuse and CD predicted adult antisocial behavior when covariates were controlled. Further analysis indicated that early alcohol abuse significantly mediated but did not moderate the effect of $\mathrm{CD}$ on antisocial behavior.

While results of both these studies concur in suggesting that alcohol abuse in the early teens may play a causal role as a risk factor in the etiology of adult antisocial behavior, they are not sufficient to definitively demonstrate this since both studies were cross-sectional in design and relied on retrospective reporting of $\mathrm{CD}$, alcohol abuse and antisocial behavior. However, at least two subsequent studies using a prospective design support the idea that early alcohol abuse is causally related to the subsequent development of antisocial traits and behaviors in late adolescence and early adulthood. The first [17] examined the bidirectional association between changes in alcohol use and psychopathic traits - interpersonal, callous-unemotional and impulsiveirresponsible - across 7 annual assessments in a large sample of serious male adolescent offenders (ages 17 - 23). The focus was on disaggregating between- and within-person changes. Several findings are of particular relevance to the Howard hypothesis. First of all, regarding between-person associations, individuals having higher overall levels of psychopathy also displayed greater overall levels of alcohol use. Evidence especially pertinent to Howard's hypothesis was obtained when within-person associations were examined. First, greater use of alcohol at any time point was associated with a higher level of psychopathic traits. More importantly, higher than expected levels of alcohol use at any one time point were associated with higher than expected psychopathic features at subsequent time points. This clearly, in accord with Howard's hypothesis, implicates excessive alcohol use in adolescence as a causal risk factor in the development of adult psychopathic personality features. Important to note here is the well-established relationship between psychopathic personality features and violent offending (reviewed in [18]). The second study [19] examined alcohol use and antisocial behavior measured at 12, 13, 15 and 17 years of age in a large UK community cohort of male and female adolescents who were followed prospectively. Importantly, although bidirectional relations were found between alcohol use and antisocial behavior, alcohol use was predictive of antisocial behavior in late adolescence (between ages 15 and 17), particularly in males. While these studies concur in supporting excessive alcohol use as a causal risk factor for antisocial behavior, at least in males, the clearer pattern of results found in the first study is likely attributable to its sample comprising male offenders, in contrast with the second sample that comprised male and female community residents. 


\section{Emotional impulsiveness is a core feature of severe personality disorder}

Emotional impulsiveness refers to the tendency to act rashly and intemperately under the pressure of positive or negative emotions [20]. When behaving in an emotionally impulsive way, the individual responds to a stimulus or event on the basis of an immediate emotional reaction such as desire or anger, with little or no checking of long-term consequences [21]. While most self-report measures of impulsiveness lack a clear and explicitly emotional component, a recently developed model of impulsive behavior, derived from the Five Factor Model of normal personality, conceptualizes and assesses impulsiveness as a multifaceted construct that includes four distinct and separable pathways to impulsive behavior: Urgency, (lack of) Premeditation, (lack of) Perseverance and Sensation seeking (hence it is commonly referred to by its acronym "UPPS"). The Urgency scale clearly and explicitly reflects negative affectivity, measuring "a tendency experience strong impulses, frequently under conditions of negative affect" [22, p. 685]. Subsequently, UPPS was revised ("UPPS-P") to include a positive Urgency scale to reflect impulsive behavior occurring in the context of positive affect [23]. A recent meta-analytic review [24] of the psychopathological correlates of UPPS scales revealed that the Negative Urgency scale demonstrated the greatest correlational effect sizes across the various forms of psychopathology examined, including alcohol/substance abuse, depression, suicidality/nonsuicidal self-injury, aggression, anxiety, borderline personality disorder, and eating disorder. Positive Urgency demonstrated a pattern of correlations similar to that of Negative Urgency, leading the authors to suggest that Positive and Negative Urgency may be “...separate but closely related "subprocesses" of a broader dimension implicating strong emotion, regardless of valence, and impulsive action in response to that emotion" [24, p. 9]. From this meta-analytic review it can be concluded that emotional impulsiveness (Urgency) appears to be associated with a broad range of both internalizing and externalizing psychopathologies, and to reflect the intersection of internalizing and externalizing tendencies seen in severe personality disorder. Recent reports of studies within the personality disorder literature, e.g. [25], suggest a higher-order general (g) factor reflecting overall severity of personality disorder that cuts across different diagnostic categories and reflects both internalizing and externalizing processes, as well as cognitive disturbance (odd beliefs, ideas of reference).

A recent study of personality disordered offenders [26] confirmed that severity of personality disorder measured either by combining externalizing and externalizing features or by aggregating across dimensional scores of individual personality disorders, was associated with a high level of negative Urgency. Of all the UPPS scales, only Urgency was able to predict severity of personality disorder. Results were consistent with those of a previous study of predominantly female patients with personality disorder [27]. This study showed that Urgency correlated with 3 of the 5 trait domains included in the DSM-5 [28] hybrid model: Negative Affectivity, Antagonism and Disinhibition) and with
14 of 25 lower-order traits. In short, therefore, a high level of emotional impulsiveness is associated with greater severity of personality disorder as indexed by a greater co-occurrence of different personality disorder categories or by the confluence of externalizing and internalizing features.

Severe personality disorder (and hence also emotional impulsiveness) is associated with severe violence

Evidence supports the idea that emotional impulsiveness, as a core feature of severe personality disorder, contributes to an increased risk of violence. In a study of personality disordered offenders, Urgency correlated significantly with a composite measure of serious violence comprising serious violence in the criminal record, early onset of violent behaviour and serious institutional violence [29]. In essentially the same sample, severity of personality disorder, indexed by summing across personality disorder symptoms, correlated significantly with the degree of severe violence in patients' criminal records and with a younger age at onset of violent offending [30]. Urgency also appears to be a potentially useful measure of risk of future violence. In a study of predictors of violence in psychiatric inpatients, negative Urgency in combination with having a personality disorder predicted both the number of weeks in which physical aggression was observed by psychiatric nurses as well as physical aggression within the first 12 weeks of admission [31].

Reoffending in PD patients released from secure care into the community is associated with a toxic triad: severe drug and alcohol abuse, adult antisocial personality with severe $C D$, and severe borderline PD

A study conducted by the current author and colleagues [32] examined the ability of a composite risk measure to predict reoffending in 53 personality disordered male patients following their release into the community. By the end of the $5^{\text {th }}$ year after discharge from medium security, just over half the men had reoffended. The mean time to reconviction was just over 2 years. The risk instrument comprised 3 elements, each scored on a scale of $0-2$ :

1. Severity of antisocial personality disorder, where a score of 2 captured severe CD (10 or more DSM-IV CD criteria met), while a score of 0 was indicated by the absence of both child and adult antisocial criteria;

2. Severity of borderline personality disorder, where a score of 2 captured severe borderline symptomatology ( 7 or more DSM-IV criteria met), while a score of 0 indicated an absence of borderline symptoms;

3. Substance dependence prior to admission, where a score of 2 indicated both drug and alcohol dependence, while a score of 0 indicated the absence of both drug and alcohol dependence.

Results supported the ability of the tripartite risk measure to predict reoffending. Each of the 3 elements of the risk measure, as well as the scale total, correlated highly and significantly 
with the second factor (Factor 2) of the Psychopathy ChecklistRevised(PCL-R: [33]) which captures deviant and disinhibited behavior from a young age. No association however was found between the tripartite risk measure and core interpersonal and affective features of psychopathy. These results suggest that when antisocial and borderline features of personality disorder co-occur with a history of severe childhood CD and substance dependence, the risk of reoffending is greater than in the absence of these features.

\section{Emotional impulsiveness as seen in severe personality disorder commonly manifests in anger}

Perhaps the most common concomitant of emotional impulsiveness is the experience and expression of anger. In a recent study of the primary emotions experienced by patients with personality disorder [34], anger ("feeling hotheaded, being easily irritated and frustrated, experiencing anger verbally or physically and remaining angry for long periods") emerged as the emotion that was most clearly linked to personality disorder across most categories. The experience of anger was significantly associated with paranoid, borderline, antisocial, narcissistic and obsessive-compulsive personality disorders.

A study by the current author and colleagues [35] found that among treatment-seeking patients with personality disorders, those who met both adult criteria for antisocial personality disorder and criteria for borderline personality disorder showed significantly greater severity of CD when compared with those showing just adult features of antisocial personality disorder. The comorbid group also showed significantly greater levels of both state (outwardly expressed) and trait anger compared with the adult antisocial alone group, as well as greater personality disorder comorbidity in general, i.e. greater overall severity of personality disorder.

A recent prospective, longitudinal study of very early (age 14 - 36 months) antecedents of childhood conduct disorder found that antisocial propensity could be identified during toddlerhood [36]. Disregard for others (responding to others' distress with anger and hostility) was a robust predictor of conduct problems in later childhood and adolescence. Other significant predictors were general cognitive ability and language ability.

Another recent propspective study [37] delineated trajectories of anger among 503 boys using annual assessments from childhood to middle adolescence (ages 7 - 14). Associations between these trajectories and features of antisocial personality in young adulthood (age 28) were examined, including whether cognitive control moderates this association. As well as demonstrating considerable heterogeneity in the developmental course of anger among boys, findings from this study suggested that youth who exhibited a pattern of chronic anger beginning in childhood were at particularly high risk for displaying antisocial personality features in adulthood, but only when coupled with poor cognitive control assessed during adolescence.

Taken together, results of these studies suggest that the propensity to experience and express anger may be an important strand that runs continuously through the developmental fabric of severe personality disorder, from early childhood through late childhood and adolescence, to adulthood. When anger issues in violence,it may be the most significant manifestation of emotional impulsiveness. In a study of the functions of violence perpetrated by young men under the influence of alcohol [38], it was found that their violence, both when it was impulsive motivated by a desire to remove an immediate threat - and when it was premeditated - motivated by a desire for revenge -served the function of anger expression.

\section{An emotionally impulsive brain can be trained to be less impulsive}

Emotional impulsiveness is arguably a dynamic, rather than static, risk factor for violence; that is to say, it is changeable. If this is the case, it should be possible to train an emotionally impulsive individual, through the use of biofeedback, to alter his pattern of brain activity in a non-impulsive direction. This was attempted in a patient who showed both borderline and antisocial personality disorder features [39]. Both self-report and electrophysiological (Go/No Go CNV: see Fig. 1) measures of impulsiveness were taken before and following several weeks of biofeedback training in which the patient was trained, successfully, to control his slow brain potentials (see [39] for details). Although these results are preliminary and restricted to a single patient, they are promising in demonstrating that emotional impulsiveness is changeable. It remains to be demonstrated that reducing emotional impulsiveness in this way can reduce the risk of reoffending.

\section{Some unresolved issues}

As outlined above, evidence has accrued supporting the hypothesis proposed by the current author since it was first advanced some 10 years ago. However, some major gaps remain. In particular, evidence is lacking that supports the proposed mechanisms by which, first, excessive alcohol use is initiated in a proportion of those with CD; and second, excessive alcohol use in early/mid adolescence translates into later deficits in emotional self-regulation. These proposed mechanisms are, respectively, an impaired neural socio emotional system and an impaired neural cognitive control system. While it is well documented that excessive alcohol use, particularly binge drinking, results in neuropsychological impairment in adolescents[40], we currently lack behavioral, neuropsychological and criminological data from prospective studies that follow alcohol abusing and non-abusing individuals longitudinally from early through late adolescence and into young adulthood. We also lack data that would confirm the hypothesis that the (roughly) one-third of children with CD who show co-occurring callous and unemotional (CU) traits overlap with those who show early-onset alcohol abuse. Since CU traits are thought to be associated with a reward oriented response set and thrill-seeking fearlessness [41], It would be reasonable to suppose that young adolescents with these traits would be more inclined to engage in early use and abuse of alcohol (as well as other drugs) as part of their thrill-seeking behavioral repertoire. However, this remains, to the author's knowledge, to be verified. If this turned out to be the case it would imply that the 
well documented association between CU traits and a particularly stable, severe and aggressive pattern of antisocial behavior [41] might be explained, at least in part, by the effects of excessive alcohol use on the developing adolescent brain.

\section{Conclusion}

Since its original formulation, and its later elaboration [12] Howard's hypothesis has generated research findings that are largely consistent with its main propositions. However, parts of the hypothesis remain to be tested, in particular those parts of the hypothesis that propose a mediating role for changes brought about by adolescent alcohol abuse in the socioemotional and cognitive control systems of the brain.

\section{Declarations}

The author declares no conflicts of interest. Studies conducted by the author and his co-workers described herein all received ethical approval from the North Nottinghamshire Research Ethics Committee.

\section{References}

1. Howard RC. How is personality disorder linked to dangerousness? A putative role for early-onset alcohol abuse. Medical Hypotheses. 2006;67(4):702-708. DOI:10.1016/j.mehy.2006.03.050.

2. Neo LH, McCullagh P, Howard RC. An electrocortical correlate of a history of alcohol abuse in criminal offenders. Psychology, Crime and Law. 2001;7(1-4):105-117. Doi:10.1080/10683160108401790.

3. Howard RC, Lumsden J. A neurophysiological predictor of re-offending in Special Hospital patients. Criminal Behaviour and Mental Health. 1996;6(2):147-156. DOI:10.1002/cbm.82

4. Howard RC, Lumsden J. CNV predicts violent outcomes in patients released from special hospital. Criminal Behaviour and Mental Health. 1997;7(3):237-240. DOI:10.1002/cbm.176.

5. Kjelsberg E. Exploring the link between conduct disorder in adolescence and personality disorders in adulthood. Psychiatric Times. 2006;23(8):22-24.

6. Farrington DP, Ttofi MM, Coid JW. Development of adolescence limited, late-onset, and persistent offenders from age 8 to age 48 . Aggressive Behavior. 2009;35(2):150-163. doi:10.1002/ab.20296.

7. Wells JE, Horwood LJ, Fergusson DM. Drinking patterns in mid adolescence and psychosocial outcomes in late adolescence and early adulthood. Addiction. 2004;99(12):1529-1541. D0I:10.1111/j.13600443.2004.00918.x.

8. Salas-Wright CP, Reingle Gonzalez JM, Vaughn MG, Schwartz SJ, Jetelina KK. Age-related changes in the relationship between alcohol use and violence from early adolescence to young adulthood. Addictive Behaviors Reports. 2016;4:13-17. Doi:10.1016/j.abrep.2016.05.004.

9. Burke JD, Waldman I, Lahey BB. Predictive validity of childhood oppositional disorder and conduct disorder: Implications for the DSM-V. Journal of Abnormal Psychology. 2010;119:739-751. doi:10.1037/a0019708. doi:10.1037/a0019708.

10. Robins LN. Sturdy childhood predictors of antisocial behaviour: Replications from longitudinal studies. Psychological Medicine. 1978;8(4):611-622.

11. Myers MG, Stewart DG, Brown SA. Progression from conduct disorder to antisocial personality disorder following treatment for adolescent substance abuse. American Journal of Psychiatry. 1998;155(4):479_

\section{DOI:10.1176/ajp.155.4.479}

12. Howard RC, McMurran M. Alcohol and violence in developmental perspective. In McMurran $M$, editor. Alcohol-Related Violence: Prevention and Treatment, Chichester: Wiley-Blackwell; 2013;81102.

13. Steinberg L. A social neuroscience perspective on adolescent risk taking. Developmental Review. 2008;28(1):78-106. doi:10.1016/j. dr.2007.08.002

14. Shulman EP, Smith AR, Silva K, Icenogle G, Duell N, Chein J, et al The dual systems model: review, reappraisal and reaffirmation. Developmental Cognitive Neuroscience. 2016;17:103-117. doi:10.1016/j.dcn.2015.12.010

15. Howard RC, Finn PR, Gallagher J, Jose PE. Adolescent-onset alcohol abuse exacerbates the influence of childhood conduct disorder on late adolescent and early adult antisocial behaviour. Journal of Forensic Psychiatry \& Psychology. 2012;23:7-22. doi:10.1080/14789949.201 1.641996.

16. Khalifa N, Duggan, C, Howard, R, Lumsden J. The relationship between childhood conduct disorder and adult antisocial behavior is partially mediated by early-onset alcohol abuse. Personality Disorders: Theory, Research, and Treatment. 2012;3(4):423-432. doi:10.1037/ a0027017.

17. Hawes SW, Crane CA, Henderson CE, Mulvey EP, Schubert CA, Pardini DA. Codevelopment of psychopathic features and alcohol use during emerging adulthood: Disaggregating between- and within-person change. Journal of Abnormal Psychology. 2015;124(3):729-739. Doi:10.1037/abn0000075.

18. Howard RC. Personality disorders and violence: what is the link? Borderline Personality Disorder and Emotion Dysregulation. 2015;2:12. DOI:10.1186/s40479-015-0033-x

19. Cho S-B, Heron J, Aliev F, Salvatore JE, Lewis G, Macleod J, et al. Directional relationships between alcohol use and antisocial behavior across adolescence. Alcoholism: Clinical and Experimental Research. 2014;38(7):2024-2033. DOI:10.1111/acer.12446.

20. Shapiro D. Neurotic Styles. New York: Basic Books. 1965.

21. Wingrove J, Bond AJ. Impulsivity: a state as well as trait variable. Does mood awareness explain low correlations between trait and behavioural measures of impulsivity? Personality and Individual Differences. 1997;22(3):333-339. doi:10.1016/S01918869(96)00222-X.

22. Whiteside SP, Lynam DR. The five factor model and impulsivity: using a structured model of personality to understand impulsivity. Personality and Individual Differences. 2001;30(4):669-689. DOI:10.1016/S0191-8869(00)00064-7.

23. Lynam D, Smith G, Whiteside S, Cyders M. The UPPS-P: Assessing five personality pathways to impulsive behavior (technical report);2006. West Lafayette, IN: Purdue University.

24. Berg JM, Latzman RD, Bliwise NG, Lilienfeld SO. Parsing the heterogeneity of impulsivity: a meta-analytic review oft he behavioral implications of the UPPS for psychopathology. Psychological Assessment. 2015;27(4):1129-1146.

25. Sharp C, Wright AGC, Fowler JC Frueh BC, Allen JG, Oldham J, et al. The structure of personality pathology: both general (' $\mathrm{g}$ ') and specific ('s') factors? Journalof Abnormal Psychology. 2015;124(2):387-398. doi:10.1037/abn0000033.

26. Howard R, Khalifa N. Is emotional impulsiveness (Urgency) a core 
feature of severe personality disorder? Personality and Individual Differences. 2016;92:29-32. Doi:10.1016/j.paid.2015.12.017

27. Few LR, Lynam DR, Miller JD. Impulsivity-related traits and their relation to DSM-5 section II and III personality disorders. Personality Disorders. 2015;6(3):261-266. doi:10.1037/per0000120.

28. American Psychiatric Association [APA]. Diagnostic and statistical manual of mental disorders. 5th edition. Arlington, VA: American Psychiatric Publishing; 2013.

29. Howard RC, Khalifa N, Duggan C. Antisocial personality disorder comorbid with borderline pathology and psychopathy is associated with severe violence in a forensic sample. Journal of Forensic Psychiatry and Psychology. 2014;25:658-672. DOI:10.1080/147899 49.2014.943797.

30. Howard RC, Hepburn E, Khalifa N. Is delusional ideation a critical link in the nexus between personality disorder and violent offending? Journal of Forensic Psychiatry and Psychology. 2015;26(3):368-382. doi:10.1080/14789949.2015.1017594.

31. Bousardt AMC, Addriaan W, Hoogendoorn AW, Noorthoorn EO, Hummeln JW, Nijman HLI. Predicting inpatient aggression by selfreported impulsivity in forensic psychiatric patients. Criminal Behaviour and Mental Health. 2015;26(3):161-173. Doi:10.1002/ cbm.1955.

32. Howard R, McCarthy L, Huband N, Duggan C. Re-offending in forensic patients released from secure care: the role of antisocial/borderline co-morbidity, substance dependence, and severe childhood conduct disorder. Criminal Behaviour and Mental Health. 2013;23(3):191202. doi:10.1002/cbm.1852.

33. Hare RD. The Hare psychopathy checklist. 2nd ed. Toronto: MultiHealth Systems; 2003.
34. Karterud, S, Pedersen G, Johansen M, Wilberg T, Davis K, Panksepp J. Primary emotional traits in patients with personality disorders. Personality and Mental Health. 2016; doi:10.1002/pmh.1345.

35. Howard RC, Huband N, Duggan C. Adult antisocial syndrome with co-morbid borderline pathology: association with severe childhood conduct disorder. Annals of Clinical Psychiatry. 2012; 24: 127-134.

36. Ree SH, Friedman NP, Corley RP, Hewitt JK, Hink LK, Johnson DP, et al. An examination oft he developmental propensity model of conduct problems. Journal of Abnormal Psychology. 2016;125(4):550-564. doi:10.1037/abn0000128.

37. Hawes SW, Perlman SB, Byrd AL, Raine A, Loeber, R, Pardini DA Chronic anger as a precursor to adult antisocial personality features: the moderating influence of cognitive control. Journal of Abnormal Psychology. 2016;125(1):64-74. doi:10.1037/abn0000129.

38. Howard RC, Howells K, Jinks M, McMurran MA quadripartite typology of violence (QTV): Relationships with functions of aggression in violent youths. In: Needham I, Callaghan P, Palmstierna T, Nijman $\mathrm{H}$, Oud N, editors. Violence in clinical psychiatry. The Netherlands: Kavanah; 2009;342-345.

39. Howard RC, Schellhorn K, Lumsden J. Complex case: A biofeedback intervention to control impulsiveness in a severely personality disordered forensic patient. Personality and Mental Health. 2013;7(2):168-173. DOI:10.1002/pmh.1231.

40.Witt ED. Research on alcohol and adolescent brain development: opportunities and future directions. Alcohol. 2010;44(1):119-124. doi:10.1016/j.alcohol.2009.08.011.

41. Frick PJ, White SF. Research review: the importance of callousunemotional traits for developmental models of aggressive and antisocial behaviour. Journal of Child Psychology and Psychiatry. 2008;49(4):359-375. Doi:10.1111/j.1469-7610.2007.01862.x. 